\title{
Ecological Status near thermal power plant and jetty in Abdasa Taluka, Dist- Kutch Gujarat India
}

\begin{abstract}
The thermal power plant has major impact of hot water discharge in to nearby creek or into sea on its ecosystem. The baseline for avian biodiversity, marine ecosystem and terrestrial ecosystem has to be assessed before discharging the hot water into sea. We have assessed the terrestrial as well as marine ecosystem for baseline scenario near the thermal power plant in Abdasa taluka, Kutch Gujarat. In the present study, we have focused on terrestrial baseline status of ecological system of surrounding. The findings state that area is not rich in biodiversity but mangrove biodiversity and density may be affected due to the thermal power plant activities.
\end{abstract}

Keywords: Avian biodiversity, mangrove, hot water discharge, impact, endangered species, thermal power plant
Volume 3 Issue 5 - 2018

\author{
Ashok K Rathoure \\ Ecology \& Biodiversity Expert, Eco Chem Sales \& Services; \\ Ecosystem Resource Management Pvt. Ltd., India
}

Correspondence: Ashok K Rathoure C/O Mr. Gyanendra K Rathoure, Mayashivraj Sadan, Gupta Colony, Hardoi-24I00I, (UP), India, Tel +9l 945050 I47I, Email asokumr@gmail.com

Received: August 26, 2018 | Published: October 02, 2018

\section{Introduction}

The area in question has one thermal power plant running by Sanghi Industries Limited and one Jetty (walkway accessing the centre of an enclosed water body or structure that projects from the land out into water) structure built in 1994-1995 which is mainly used to Cement and other solid cargo handling by Sanghi Industries Limited. Sanghi Industries Limited is among leading cement manufacturers from Western India.

Water bodies should have the biological and chemical characteristics expected under sustainable conditions. The degree of departure of current vegetation from the potential natural vegetation, or potential natural community has detrimental impact on ecological system of the area. Before detailing the study area, a cursory understanding of the state scenario is important. Biodiversity of this region is largely related to the Thar Desert. Fossiliferous Limestone was found (Nummulites) which were mainly Marine deposits (coin-shaped) in

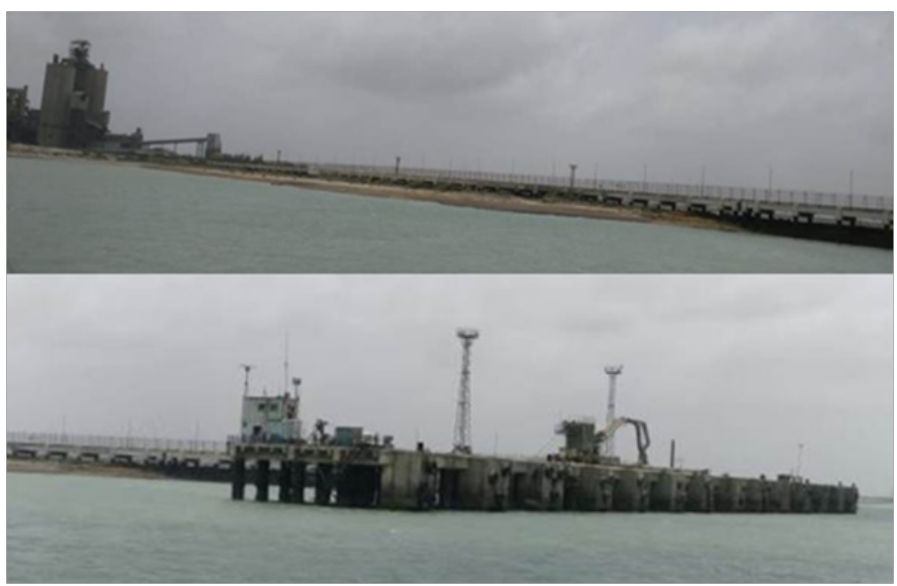

Figure I Jetty Structure. this area. Due to unfavourable meteorological conditions and diverse habitat, a rapid snapshot survey for biological reconnaissance was conducted in this case. Secondly, the activity rhythms of different species differ on a diurnal scale. For instance the rodents dwelling in the sandy tracts of the buffer zone were seen despite being ubiquitous, but leave unmistakable sole imprints of the hind paws on the sand. Such indirect species specific evidences of the animals' occupancy of the habitat have been considered. With desertic conditions, coastal vicinity, open scrub forest and slight undulating terrain in this region provides a unique ecosystem. The adverse climate and edaphic conditions intermixed with saline open plains and mangrove forests along the coast have turned this desert into an ideal dwelling place for wildlife and birds. Though forest areas are open, scrubby and along the coast they are of saline nature, some of the most endangered and threatened species of animals and birds survive in this area. As the district lies in poor rainfall area it lacks the richer mammalian life, but has the two form of wildlife are terrestrial and avifauna (Figure 1\&2).

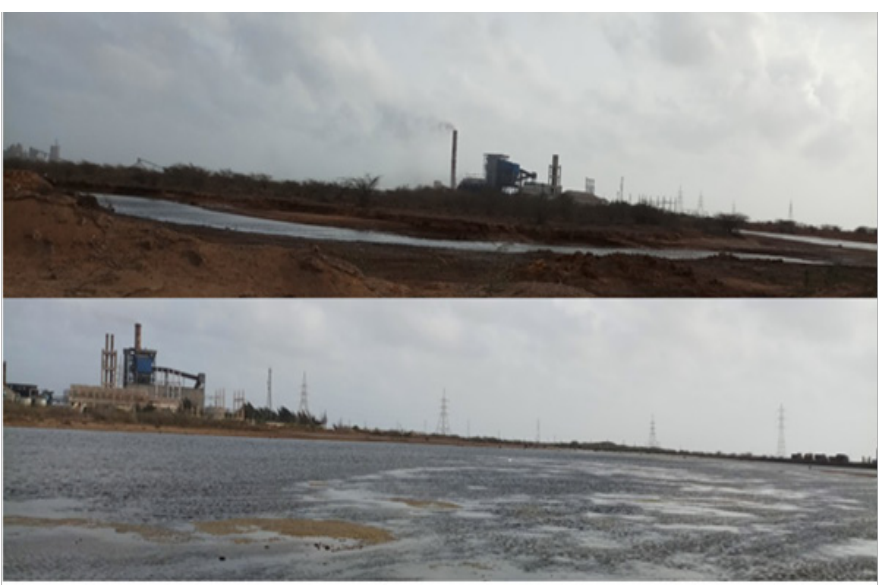

Figure 2 Thermal power plant. 


\section{Methodology}

An ecological survey of the study area was conducted particularly with reference to listing of species and assessment of the existing baseline ecological conditions in the study area. Total 8 hotspots/ villages has been identified in the study area are listed in Table 1 . The methodology adopted for the survey is depicted in Table 2.

Table I List of villages/spots of survey

\begin{tabular}{lll}
\hline SNo & Name of Village/Spots & Remarks \\
\hline I & Akari & $\begin{array}{l}\text { A small village nearest to project site, about } 22 \text { families residing in the village, very } \\
\text { few agriculture fields are there to grow the crops which are rain dependent. } \\
\text { The locals in these village practicing agriculture and milk. The Moti Ber village is the } \\
\text { biggest in the area having more than } 600 \text { families. }\end{array}$ \\
3 & Moti Ber & Village having 40 families practicing the agriculture. \\
4 & Navavas & Small village practicing agriculture and milk \\
5 & Thumdi & Open jungle of Prosopis juliflora and Acacia nilotica on SW with scattered \\
6 & Golay RF & agriculture fields. \\
7 & Walawadi & A very small village of 22 families, some agriculture fields \\
8 & West Mangrove Forest & Mangrove Forest in coastal area on SE direction. \\
\hline
\end{tabular}

Table 2 Mode of data collection and parameters considered during the survey+

\begin{tabular}{|c|c|c|c|c|c|}
\hline \# & Aspect & Data & Mode of Data Collection & Parameters Monitored & Remarks \\
\hline $\mathrm{I}$. & $\begin{array}{l}\text { Terrestrial } \\
\text { Ecology }\end{array}$ & $\begin{array}{l}\text { Primary data } \\
\text { collection }\end{array}$ & By Field Survey ${ }^{1-6}$ & $\begin{array}{l}\text { For Floral diversity, Vegetation } \\
\text { measurements: } \\
\text { Tree, } \\
\text { Shrub, } \\
\text { Herbs, } \\
\text { Grasses, } \\
\text { Climbers, } \\
\text { Cultivated plants in the study area, } \\
\text { Floristic composition of the study } \\
\text { area, } \\
\text { Medicinal plants of the study area, } \\
\text { Status of the forest, their category in } \\
\text { the study area, } \\
\text { Rare and endangered flora in the } \\
\text { study area. } \\
\text { Endemic plants in the study area. } \\
\text { For Fauna in the study area: } \\
\text {-Reptiles, } \\
\text {-Amphibians, } \\
\text {-Birds, } \\
\text {-Fresh water fishes } \\
\text {-Mammals, } \\
\text {-Butterflies. } \\
\text { Rare and Endangered fauna in the } \\
\text { study area, } \\
\text { Endemic fauna in the study area, } \\
\text { Wild life and their conservation } \\
\text { importance in the study area. }\end{array}$ & $\begin{array}{l}\text { Random survey, opportunistic } \\
\text { observations, diurnal bird } \\
\text { observation, active search } \\
\text { for reptiles, faunal habitat } \\
\text { assessment, active search } \\
\text { for microhabitat, scats, foot } \\
\text { prints, animal call, pug marks, } \\
\text { debarking sign, Nesting, Claws, } \\
\text { Dung, etc. and information } \\
\text { from local villagers. }\end{array}$ \\
\hline 2. & & $\begin{array}{l}\text { Secondary } \\
\text { data } \\
\text { collection }\end{array}$ & $\begin{array}{l}\text { Kutchh SF Division under SF Circle Bhuj. } \\
\text { Data of Fisheries department. } \\
\text { Literature like research papers, books } \\
\text { published by research/academic } \\
\text { Institutions. } \\
\text { Reports (Research reports, previous EIA } \\
\text { reports etc.) }\end{array}$ & $\begin{array}{l}\text { Interpretation of secondary data for } \\
\text { Ecological Sensitive Areas such as } \\
\text { national forests, wild life sanctuaries, } \\
\text { lakes, ravines, hills, hillocks and } \\
\text { reserve forest, vegetation, type, } \\
\text { importance etc. }\end{array}$ & $\begin{array}{l}\text { The literature was collected } \\
\text { from various authentic } \\
\text { sources. }\end{array}$ \\
\hline
\end{tabular}


Table Continued........

\begin{tabular}{llll}
\hline \# Aspect & Data & Mode of Data Collection & Parameters Monitored \\
\hline & & Wild life importance, \\
& & Floral Endemicity, \\
& & Faunal Endemicity, \\
& & State of Terrestrial vegetation, \\
& & State of wet land vegetation, \\
& & Mangrove vegetation, \\
Evaluation & & Conservation importance, \\
Ecological & Secondary & Review and Discussion & Legal status (National park, Wild life \\
& & sanctuary, Reserve forest, Wetlands, \\
& & Agricultural lands) \\
& & Lakes /reservoirs/dam, \\
& & Natural lakes and Swamps, Breeding \\
& & ground of Migratory and Residential
\end{tabular}

\section{Results and discussion}

Kutch bhuj district is forefront in agriculture field. The crops like groundnut, till, bajri etc. are taken in monsoon. The agriculture is rain dependent. No means of major surface water available for crop irrigation. The area falls under agro-climatic zone XIII as per IASRI

http://www.iasri.res.in/agridata/12data/chapter1/db2012tb1_2. pdf.

\section{Floral diversity of the study area}

The climatic, edaphic and biotic variations with their complex interrelationship and composition of species, which are adapted to these variations, have resulted in different vegetation cover, characteristic of each region..$^{20}$ The tree species, herbs, shrubs, climbers and major crops, were documented during this base line study. ${ }^{21-24}$

Trees: The dominant trees in the study area are Mangifera indica $L$ (Mango trees) Azadirachta indica (Neem), Plumeria rubra L. (Champa), Babool (Acacia nilotica), Casuarina equisetifolia L. (Casuarina), Dalbergia sisoo L. (Shisham), etc. Total 34 species of trees belong to 17 families are enumerated from the study area (Table $3)$.

Table 3 List of trees in the study area

\begin{tabular}{lll}
\hline S.No. & Family and Scientific name & Vernacular name \\
\hline I & Anacardiaceae & Keri \\
I/I & Mangifera indica $L$ & \\
$\mathbf{2}$ & Annonaceae & Asopalav \\
$2 / I$ & Polyalthia longifolia $L$. & \\
$\mathbf{4}$ & Apocynaceae & Champa \\
$3 / /$ & Plumeria rubra $L$. & Imli \\
$4 / 2$ & Tamarindus indica $L$. & \\
$\mathbf{5}$ & Burseraceae & Guggal \\
$5 / I$ & Commiphora wightii $L$. & \\
$\mathbf{6}$ & Casuarinaceae & Sharu \\
$6 / I$ & Casuarina equisetifolia $L$. & \\
$\mathbf{7}$ & Caricaceae & Papaya \\
$7 / I$ & Carica papaya L. & \\
\hline
\end{tabular}

Table Continued........

\begin{tabular}{|c|c|c|}
\hline S.No. & Family and Scientific name & Vernacular name \\
\hline 8 & Euphorbiaceae & \\
\hline $8 / 1$ & Emblica officinalis $L$. & Anola \\
\hline 7 & Fabaceae & \\
\hline $9 / 1$ & Dalbergia sisoo $L$. & Sisso \\
\hline $10 / 2$ & Delonix regia (Boj) & Gulmohar \\
\hline $11 / 3$ & Parkinsonia aculeate L. & Rambaval \\
\hline $12 / 4$ & Peltophorum pterocarpum (DC.) & Tamrafal \\
\hline $13 / 5$ & Albizia lebbeck L. & Siris \\
\hline $14 / 6$ & Albizia procera $L$. & Safed Siris \\
\hline 8 & Leguminosae & \\
\hline $15 / 1$ & Derris indica (Lam.) & Karanj \\
\hline $16 / 2$ & Parkinsonia aculeata $L$. & Vilayati Kikar \\
\hline 9 & Lythraceae & \\
\hline $17 / 1$ & Sonneratia apetala $L$. & Blume Mangrove \\
\hline $18 / 2$ & Sonneratia alba $L$. & Mangrove Apple \\
\hline 10 & Meliaceae & \\
\hline $19 / 1$ & Azadirachta indica A.Juss & Limdo \\
\hline I I & Mimosaceae & \\
\hline $20 / 1$ & Acacia nilotica $L$. & Desi Baval \\
\hline $21 / 2$ & Leucaena leucocephala (Lam.) De & Pardesi Baval \\
\hline $22 / 3$ & Pithecellobium dulce (Roxb.) Bth. & Jungle jalebi \\
\hline $23 / 4$ & Prosopis cineraria (L.) & Khejari \\
\hline $24 / 5$ & Acacia senegal (L.) Willd, & Baval \\
\hline 12 & Moraceae & \\
\hline $25 / 1$ & Ficus benghalensis L. & Bargad \\
\hline $26 / 2$ & Ficus religiosa L. & Pipal \\
\hline 13 & Moringaceae & \\
\hline $27 / 1$ & Moringa oleifera Lam & Sargavo \\
\hline 14 & Myrtaceae & \\
\hline $28 / 1$ & Eucalyptus citriodora $\mathrm{Hk}$. & Nilgari \\
\hline
\end{tabular}


Table Continued.......

\begin{tabular}{lll}
\hline S.No. & Family and Scientific name & Vernacular name \\
\hline $29 / 2$ & Syzygium cumini L. & Jambu \\
$\mathbf{1 5}$ & Rhamnaceae & \\
$30 / 1$ & Zizyphus mauritiana & Bor \\
$31 / 2$ & Zizyphus xylopyrus L. & Jungli Bor \\
16 & Salvadoraceae & \\
$32 / 1$ & Salvadora persica L. & Piludo \\
$33 / 2$ & Salvadora oleoides Decne. & Toothbrush Tree \\
17 & Sapotaceae & \\
$34 / 1$ & Manilkara zapota I. van Royen & Chikoo
\end{tabular}

Shrubs and Herbs: Total 20 shrub species belong to 15 families are enumerated from the study area. The dominant shrub community in this area was represented by Prosopis juliflora, Calotropis procera, Cereus peruvianus, Xanthium strumarium L., Abelmoschus manihot L., Hibiscus rosa sinensis L., etc. The shrubs observed in the study area are given in the Table 4 and herbs encountered in the study area are listed in Table 5.

Table 4 List of shrubs in the study area

\begin{tabular}{|c|c|c|}
\hline S.No. & Family and Scientific name & $\begin{array}{l}\text { Vernacular } \\
\text { name }\end{array}$ \\
\hline I & Apocynaceae & \\
\hline $1 / I$ & Thevetia peruviana & Pili Kaner \\
\hline 2 & Asclepiadaceae & \\
\hline $2 / 1$ & Calotropis procera & Akoda \\
\hline 3 & Bignoniaceae & \\
\hline $3 / 1$ & Tecoma stans (L.) H.B. \& K. & Peilafol \\
\hline 4 & Cactaceae & \\
\hline $4 / 1$ & Cereus peruvianus & Cactus \\
\hline $5 / 2$ & Opuntia elatior Mill. & Fafdo thor \\
\hline 5 & Capparaceae & \\
\hline $6 / 1$ & Capparis decidua (Forsk) Edgew & Kerdo \\
\hline 6 & Compositae & \\
\hline $7 / 1$ & Xanthium strumarium L. & Gokhru \\
\hline 7 & Lythraceae & \\
\hline $8 / 1$ & Lawsonia inermis & Mehandi \\
\hline 8 & Euphorbiaceae & \\
\hline $9 / 1$ & Ricinus communis $L$. & Divel \\
\hline $10 / 2$ & Euphorbia nivulia Buch.-Ham & Thor \\
\hline $1 / / 3$ & Jatropha curcus L. & Ratanjot \\
\hline 9 & Malvaceae & \\
\hline $12 / 1$ & Abelmoschus manihot $\mathrm{L}$. & Jungli Bindi \\
\hline $13 / 2$ & Hibiscus rosa sinensis L. & Jasund \\
\hline 10 & Musaceae & \\
\hline $14 / 1$ & Musa paradisiaca $L$. & Kela \\
\hline
\end{tabular}

\begin{tabular}{cll} 
Table Continued...... & \\
\hline S.No. & Family and Scientific name & $\begin{array}{l}\text { Vernacular } \\
\text { name }\end{array}$ \\
\hline I I & Mimosaceae & \\
15/I & Prosopis juliflora & Gando baval \\
I2 & Nyctaginaceae & \\
16/I & Bougainvillea spectabilis Willd. & Bougainvelia \\
13 & Rhamnaceae & \\
I 7/I & Zizyphus nummularia & Jharbera \\
18/2 & Zizyphus nummularia (Burm.f.) W. & Chanibor \\
14 & Punicaceae & \\
19/I & Punica granatum & Anar \\
15 & Rutaceae & Neebu \\
$20 / 1$ & Citrus limon &
\end{tabular}

Table 5 List of herbs in the study area

\begin{tabular}{|c|c|c|}
\hline S.No. & Family and Scientific name & Vernacular name \\
\hline $\mathbf{I}$ & Asphodelaceae & \\
\hline$I / I$ & Aloe barbensis Mill. & Kunvarapato \\
\hline $2 / 2$ & Aloe vera & Kuwar Pathu \\
\hline 2 & Asteraceae & \\
\hline $3 / 1$ & Tridax procumbens $L$ & Bhangro \\
\hline $4 / 2$ & Eclipta prostrata & Bhangro \\
\hline $5 / 3$ & Echinops echinatus Roxb & Shulio \\
\hline $6 / 4$ & Lacunae procumbens (Roxb) & Moti Bhonpatri \\
\hline 3 & Convolvulaceae & \\
\hline $7 / 1$ & Cressa cretica $L$. & Palio, Rudanti \\
\hline $8 / 2$ & Ipomoea pes-carprae (L) & Dariani vel \\
\hline $9 / 3$ & Ipomoea aquatica Forsk. & Nalini Bhaji \\
\hline $10 / 4$ & Ipomoea obscura Ker & Vad fudradi \\
\hline 4 & Lamiaceae ( Labiatae) & \\
\hline 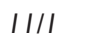 & Ocimum sanctum L. & Tulsi \\
\hline 5 & Malvaceae & \\
\hline $12 / 1$ & Abutilon indicum $L$. & Khapat, Dabaliar \\
\hline 6 & Menyanthaceae & \\
\hline $13 / 1$ & Nyphoides indicum (Roxb.) & Kumudini \\
\hline 7 & Nyctaginaceae & \\
\hline $\mid 4 / 1$ & Boerhavia diffusa $L$. & Satodi \\
\hline 8 & Papilionaceae & \\
\hline $15 / 1$ & Cortalaria medicaginea Lam & Ran methi \\
\hline 9 & Solanaceae & \\
\hline $16 / 1$ & Datura metel & Dhatura \\
\hline $17 / 2$ & Solanum nigrum $L$. & Piludi \\
\hline 10 & Zygophyllaceae & \\
\hline $18 / 1$ & Tribulus terrestris $\mathrm{L}$ & Gokhru \\
\hline
\end{tabular}


Mangrove: Some part of the study area falls coastal area which is declared as mangrove forest $(10.68 \%)$, there is Avecenia marina was observed during the study. Four species of mangrove were encountered from the study area were terrestrial mangrove (Table 6).

Table 6 List of mangrove/s encountered in the study area

\begin{tabular}{|c|c|c|c|}
\hline S.No. & Scientific name & Common name & Family \\
\hline \multicolumn{4}{|c|}{ Marine } \\
\hline I. & Avecenia marina & Grey Mangrove & Verbenaceae \\
\hline \multicolumn{4}{|c|}{ Terrestrial } \\
\hline 2. & Salvadora persica & Toothbrush Tree & Salvadoraceae \\
\hline 3. & $\begin{array}{l}\text { Salvadora oleoides } \\
\text { Decne. }\end{array}$ & Toothbrush Tree-Big & Salvadoraceae \\
\hline 4. & Sonneratia apetala & Blume Mangrove & Lythraceae \\
\hline
\end{tabular}

\section{Cultivated plants in the study area}

It is observed that, the different parts of the study area were practicing different crop pattern based on the season and availability of irrigation facility. The rain is the major source of irrigation in this area. The general crop patterns practiced in the study area were Juwar (Sorghum vulgare), Ground nut (Arachis hypogaea) and during monsoon, Wheat (Triticum aestivum) during winter and during summer only Ground nut (Mumphali) were practiced in this region.

Major horticultural crops: Plantation of Chikku (Manilkara zapota), Kela (Musa sp.) Papaya (Carica papaya), Amla (Phyllanthus emblica), Mango trees (Mangifera indica) and Dadam (Punica granatusm) were observed at some localities. Mango trees (Mangifera indica) and Tamarindus trees (Tamarindus indica) were observed adjacent to the residential area and also along the road side at almost all villages. Chikku, Aam, Amla, Limboo and Papaiyo were developed by client in the premises.

Major vegetable corps: The major vegetables grown in the study area were:

i. Bhindi (Abelmoschus escelentus),

ii. Brinjal, (Ringana Solanum melongena),

iii. Cabbage (Brassica oeraceae),

iv. Tomato (Lycopersicon lycopersicum),

v. Guvar (Cyamopsis tetragonoloba),

vi. Val (Lablab purpureus),

vii. Turia (Luffa acutangula),

viii. Karela (Momordica charantia),

ix. Drum stick (Sargva) (Moringa oleifera),

x. Amli (Tamarindus indica)

xi. Chloi (Vigna unguilata)

e. Pulses: The pulses cultivated in this region were Mag (Vigna acontifolia), Tuver (Cajanus cajan).

\section{Reet Species in the study area}

Among the enumerated flora in the study area, none of them were assigned any threat category by Red data book of Indian Plants. ${ }^{15-19,21-24}$

\section{Faunal biodiversity of the study area}

For the documentation of the faunal biodiversity of the study area with respect to birds, reptiles, amphibians, and butterfly species, a baseline survey had been conducted. The common birds observed in the study area are Accipiter badius, Halcyon coromanda, Anhinga melanogaster, Apus apus, Egretta garzetta, Columba livia, Streptopelia orientalis, Anthus campestris, Motacilla cinerea, Motacilla flava, Nectarinia asiatica, Phalacrocorax fuscicollis, Pycnonotus cafer, Limosa limosa, Platalea ajaja, etc. listed in Table 7. The Indian Peafowl was observed which is listed as schedule $-\mathrm{I}$ as per IWPA, 1972 and others listed as schedule IV as per IWPA, 1972.

Butterflies from the study area: Butterflies from three families observed during the present study are documented in the table below (Table 8).

Herpetofauna: In amphibian group, the toads were sighted during the study period. The reptile, Common Garden Lizard, House Gecko and Fan-Throated Lizard, Common rat Snake and were observed in the region is given in the table below (Table 9).

\section{Mammals}

Common Mongoose (Herpestes edwardsii), Jungle cat were observed which are protected under schedule II and Nilgai (Boselaphus tragocamelus) is Schedule-III animal as per Wildlife Protection Act 1972. The Squirrel, Indian Porcupine, Hare etc. are protected under schedule IV. The Common House Rat (Rattus rattus) is protected under schedule V (Table 10).

\section{Insect}

There is no significant faunal assemblage here except for some insects like Honey Bees (Apis sp.) and Gum leaf Grasshopper (Goniaea australasiae).

\section{Fisheries}

Gujarat is the second largest fish producing States in the country only next to West Bengal. Among all the maritime States, Gujarat accounts for a significant share insofar as marine fish production in the country is concerned and stands as the largest marine fish producer. Although, the study area have no major river or any lake, a very few fishes were observed. Some part of study area fall coastal zone, hence marine fishes reported, no fisher man or fishing activity found in the study area (Table 11), (Figure 3-5).

\section{REET faunal species}

Some of the sighted fauna was given protection by the Indian Wild Life (Protection) Act, 1972 by including them in different schedules. Among the birds in the study area, Pea fowl (Pavo cristatus) is included in schedule I of Wild life Protection Act (1972), while many other birds are included in schedule IV. Among the reptiles, Indian Cobra (Naja naja), Indian chameleon, Rat snake, Indian Krait and Indian Monitor are provided protection as per Schedule-II of Wild life Protection Act, (1972). Among mammals; Common Mongoose (Herpestes edwardsi), Indian Monitor, Jungle cat are a schedule-II animals. Nilgai (Boselaphus tragocamelus) is protected as ScheduleIII animal and hares and 5 stripped squirrels are included in schedule IV of Wild Life Protection act 1972. 
Table 7 Systematic lists of birds in the study area with status

\begin{tabular}{|c|c|c|c|c|c|}
\hline \# & Family & Scientific Name & Vernacular Name & Common Name & Status \\
\hline $\mathrm{I}$. & Accipitridae & Accipiter badius (Gmelin, I788) & Shakro & Shikra & $\mathrm{R}$ \\
\hline 2. & & Elanus caeruleus (Desfontaines, I789) & Kapasi/Laudharo & Black-winged Kite & $\mathrm{R}$ \\
\hline 3. & Alcedinidae & Halcyon coromanda (Latham, I790) & Kalkalio & Ruddy Kingfisher & $\mathrm{R}$ \\
\hline 4. & & Halcyon smyrnensis (Linnaeus, I758) & Moto Kalkalio & $\begin{array}{l}\text { White-throated } \\
\text { Kingfisher }\end{array}$ & $\mathrm{R}$ \\
\hline 5. & Anhingidae & Anhinga melanogaster (Pennant, I769) & Jalbhi & Darter & $\mathrm{R}$ \\
\hline 6. & Apodidae & Apus apus (Linnaeus, I758) & Ababil & Common Swift & $\mathrm{R}$ \\
\hline 7. & & Apus affinis (JE Gray, I830) & Moto Ababil & Little Swift & $\mathrm{R}$ \\
\hline 8. & Ardeidae & Bubulcus ibis (Linnaeus, I758) & Dhorbaglo & Cattle Egret & $\mathrm{R}$ \\
\hline 9. & & Egretta garzetta (Linnaeus, I766) & Baglo & Little Egret & $\mathrm{R}$ \\
\hline 10. & & Mesophoyx intermedia (Wagler, I827) & Vachetdholo baqlo & Intermediate Egret & $\mathrm{R}$ \\
\hline II. & Caprimulgidae & Caprimulgus asiasticus (Latham, I790) & Sonara & Nightjar & $\mathrm{R}$ \\
\hline 12. & Charadriidae & Vanellus indicus (Boddaert, I783) & Titodi & Lapwing & $\mathrm{R}$ \\
\hline 13. & Ciconiidae & Ciconia ciconia (Linnaeus, I758) & Badho & White Stork & $\mathrm{V}$ \\
\hline 14. & & Ephippiorhynchus asiaticus (Latham, I790) & Dhonk & Black necked Stork & $\mathrm{R}$ \\
\hline 15. & & Mycteria leucocephala (Pennant, I769) & Dhonk & Painted Stork & $\mathrm{R}$ \\
\hline 16. & Columbidae & Columba livia (Gmelin, I789) & Kabutar & Rock Pigeon & $\mathrm{R}$ \\
\hline 17. & & Streptopelia decaocto (Frivaldszky, 1838) & Holdi & Eurasian Collared-Dove & $\mathrm{R}$ \\
\hline 18. & & Streptopelia orientalis (Latham, I790) & Holdi & Rufous Turtle Dove & $\mathrm{R}$ \\
\hline 19. & Coraciidae & Coracias benghalensis (Linnaeus, 1758) & Deshi Neelkanth & Indian Roller & $\mathrm{R}$ \\
\hline 20. & Corvidae & Corvus splendens (Vieillot, I8I7) & Kagdo & Crow & $\mathrm{R}$ \\
\hline 21. & Cuculidae & Centropus sinensis (Stephens, I8I5) & Hoco & Coucal & $\mathrm{R}$ \\
\hline 22. & & Surniculus lugubris (Horsfield, I82I) & Kaliyakoshi & Drongo Cuckoo & $\mathrm{R}$ \\
\hline 23. & Dicruridae & Dicrurus macrocercus (Vieillot, I8I6) & Kado kosi & Black drongo & $\mathrm{R}$ \\
\hline 24. & Fringillidae & Carduelis carduelis (Linnaeus, I758) & Tapusiyu & Goldfinch & $\mathrm{R}$ \\
\hline 25. & Glareolidae & Cursorius coromandelicus (Gmelin, I789) & Rangodhlo & Courser & $\mathrm{R}$ \\
\hline 26. & Gruidae & Antigone antigone (Linnaeus, I758) & Kunj & Crane & $\mathrm{R}$ \\
\hline 27. & Laridae & Larus brunnicephalus (Jerdon, I840) & Gull & Gull brown headed & $\mathrm{R}$ \\
\hline 28. & & Sterna albifrons (Pallas, I764) & Nana vabagli & Little Tern & $\mathrm{R}$ \\
\hline 29. & Leiothrichidae & Turdoides caudate (Dumont, 1823) & Babbler & Common Babbler & $\mathrm{R}$ \\
\hline 30. & Megalaimidae & Megalaima haemacephala (Statius Muller, I776) & Kansaro & Coppersmith barbet & $\mathrm{R}$ \\
\hline 31. & Meropidae & Merops leschenaultia (Vieillot, I8I7) & Tarklo & $\begin{array}{l}\text { Chestnut-headed Bee- } \\
\text { eater }\end{array}$ & $\mathrm{R}$ \\
\hline 32. & Motacillidae & Anthus campestris (Linnaeus, I758) & Pidi dhanchidi & Tawny Pipit & $\mathrm{R}$ \\
\hline 33. & & Anthus spinoletta (Linnaeus, I758) & Panini Dhanchidi & Water Pipit & W \\
\hline 34. & & Motacilla cinerea (Tunstall, I77I) & Diwaliyo & Grey Wagtail & W \\
\hline 35. & & Motacilla flava (Linnaeus, I758) & Pilo Divaliyo & Yellow Wagtail & $\mathrm{S}$ \\
\hline 36. & Muscicapidae & Ficedula parva (Bechstein, I792) & Chatki ma khimar & Red breasted Flycatcher & $\mathrm{R}$ \\
\hline 37. & & Muscicapa striata (Pallas, I764) & Nanu Chikyu & Spotted Flycatcher & $\mathrm{S}$ \\
\hline 38. & Nectariniidae & Nectarinia asiatica (Latham, I790) & Jāmbalī Sunbird & Purple Sunbird & $\mathrm{R}$ \\
\hline 39. & & Nectarinia minima (Sykes, I832) & Motu Duriyu & Crimson-backed Sunbird & $\mathrm{R}$ \\
\hline 40. & Paridae & Cyanistes caeruleus (Linnaeus, I758) & Chikyu & Blue Tit & $\mathrm{R}$ \\
\hline $4 \mathrm{I}$. & & Parus major (Linnaeus, I758) & Tikdi & Great Tit & $\mathrm{R}$ \\
\hline
\end{tabular}


Table Continued.......

\begin{tabular}{|c|c|c|c|c|c|}
\hline \# & Family & Scientific Name & Vernacular Name & Common Name & Status \\
\hline 42. & Passeridae & Passer domesticus (Rafinesque, 1815) & Chakli & Sparrow & $\mathrm{R}$ \\
\hline 43. & Phalacrocoracidae & Phalacrocorax fuscicollis (Stephens, 1826) & Pani Kagdo & Cormorant & $\mathrm{R}$ \\
\hline 44. & Phasianidae & Francolinus pondicerianus (Gmelin, I789) & Titar & Gery Francolin & $\mathrm{R}$ \\
\hline 45. & & Pavo cristatus (Linnaeus, I758) & Mor & Indian Peafowl & $\mathrm{R}$ \\
\hline 46. & Phoenicopteridae & Phoenicopterus minor (Geoffroy Saint-Hilaire, I798) & Nano Surkabh & Lesser Flamingo & $\mathrm{R}$ \\
\hline 47. & Picidae & Picus viridis (Linnaeus, 1758) & Lakkadkhod & Green Woodpecker & $\mathrm{R}$ \\
\hline 48. & Ploceidae & Ploceus philippinus (Linnaeus, 1766) & Sugari & Baya weaver & $\mathrm{R}$ \\
\hline 49. & Podicipedidae & Tachybaptus ruficollis (Pallas, I764) & Dubki & Little Grebe & $\mathrm{R}$ \\
\hline 50. & Psittaculidae & Psittacula krameri (Cuvier, I800) & Popat & Rose-ringed Parakeet & $\mathrm{V}$ \\
\hline 51. & Pteroclidae & Pterocles exustus (Temminck, I825) & Batumdi & Sandgrouse & $\mathrm{V}$ \\
\hline 52. & Pycnonotidae & Pycnonotus cafer (Linnaeus, I766) & Bulbul & Bulbul & $\mathrm{R}$ \\
\hline 53. & Rallidae & Amaurornis phoenicurus (Pennant, I769) & Safed chatari & $\begin{array}{l}\text { White-breasted Water } \\
\text { hen }\end{array}$ & $\mathrm{R}$ \\
\hline 54. & & Fulica atra (Linnaeus, I758) & Dasadi & Common Coot & $\mathrm{R}$ \\
\hline 55. & Rallidae & Gallinula chloropus (Brisson, I760) & Jalmurgi & Moorhen & $\mathrm{R}$ \\
\hline 56. & Scolopacidae & Actitis hypoleucos (Linnaeus, I758) & Nani tutwari & Sandpiper & W \\
\hline 57. & & Limosa limosa (Linnaeus, I758) & Motagadero & Black Tailed Godwit & $\mathrm{R}$ \\
\hline 58. & & Philomachus pugnax (Linnaeus, I758) & Tilio & Ruff & $\mathrm{R}$ \\
\hline 59. & Striidae & Athene noctua (Scopoli, I769) & Nani ghuwad & Little Owl & $\mathrm{R}$ \\
\hline 60. & Sturnidae & Acridotheres ginginianus (Latham, I790) & Ghoda kabar & Bank Myna & $\mathrm{R}$ \\
\hline 61. & Threskiornithidae & Platalea leucorodia (Linnaeus, I758) & Chamchichanch & Eurasian Spoonbill & OP \\
\hline 62. & & Platalea ajaja (Linnaeus, I758) & Gulabi chamchichanch & Spoonbill & O \\
\hline 63. & & Threskiornis melanocephalus (Latham, I790) & Dhorikankansar & Black headed ibis & $\mathrm{R}$ \\
\hline
\end{tabular}

$\mathrm{R}=$ Resident $\mathrm{O}=$ occurs most of the year; $\mathrm{P}=$ Spring or autumn passage; $\mathrm{W}=$ Winter only; $\mathrm{V}=\mathrm{Vagrant} ; \mathrm{S}=$ Summer only.

Table 8 Butterflies in the study area

\begin{tabular}{|c|c|c|}
\hline Scientific Name and Family & Common Name & Relative Abundance \\
\hline \multicolumn{3}{|l|}{ Family Asclepiadaceae } \\
\hline Danaus genutia Cramer & Striped Tiger & Common \\
\hline \multicolumn{3}{|l|}{ Family Papilionidae } \\
\hline Papilio polytes & Common Mormon & Common \\
\hline \multicolumn{3}{|l|}{ Family Pieridae } \\
\hline Eurema hecabe & Common Grass yellow & Very Common \\
\hline Ixias Marianne & White orange tip & Common \\
\hline \multicolumn{3}{|l|}{ Family: Nymphalidae } \\
\hline Danaus chrysippus & Plain Tiger & Common \\
\hline Phalantha phalantha & Common Leopard & Fairy Common \\
\hline Hypolimanas misippus & Danaid egg fly & Common \\
\hline Mycalesis perseus & Common bush brown & Uncommon \\
\hline Cynthia cardui Linnaeus & Painted Lady & Uncommon \\
\hline Junonia hierta Fabricius & Yellow pansy & Common \\
\hline Junonia orithya Linnaeus & Blue pansy & Fairy Common \\
\hline
\end{tabular}


Table 9 Reptiles and amphibian in the study area

\begin{tabular}{|c|c|c|c|c|}
\hline S.No. & Family & Common Name & Scientific name & Schedule as IWPA, 1972 \\
\hline & \multirow[t]{2}{*}{ Agamidae } & Common Garden Lizard & Calotes versicolor (Cuvier, I8I7) & Not listed \\
\hline & & Fan-Throated Lizard & Sitana ponticeriana (Cuvier, I8I7) & Not listed \\
\hline & Bufonidae & Toad & Bufo bufo (Gray I825) & Not listed \\
\hline & Chamaeleonidae & Indian chameleon & $\begin{array}{l}\text { Chameleon calcaratus (Rafinesque, } \\
\text { 18I5) }\end{array}$ & Schedule II \\
\hline & Colubridae & Common Rat Snake & Ptyas mucosus (Linnaeus, 1758) & Schedule II \\
\hline & \multirow{2}{*}{ Elapidae } & Common Indian Krait* & $\begin{array}{l}\text { Bungarus caeruleus (Schneider, } \\
\text { I80I) }\end{array}$ & Schedule II \\
\hline & & Indian Cobra* & Naja naja (Linnaeus, 1758) & Schedule II \\
\hline & Gekkonidae & House Gecko & $\begin{array}{l}\text { Hemidactylus flaviviridis (Ruppell, } \\
\text { 1835) }\end{array}$ & Not listed \\
\hline & Scincidae & Brahminy Skink & Mabuya carinata (Schneider, I80I) & Not listed \\
\hline & Varanidae & Indian Monitor & Varanus bengalensis (Daudin, I802) & Schedule II \\
\hline
\end{tabular}

*Not sighted but included as per the secondary information from the villagers.

Table 10 Mammals in study area

\begin{tabular}{lllll}
\hline S.No. & Family & Common Name & Scientific name & Status as per IWPA I972 \\
\hline Antilopinae & Nilgai (Blue Bull) & Boselaphus tragocamelus (Pallas, I766) & Schedule-III \\
Felidae & Common Jungle Cat & Felis chaus (Schreber, I777) & Schedule II \\
Herpestidae & Common Mongoose & Herpestes edwardsii (É. Geoffroy Saint-Hilaire, 18I8) & Schedule II \\
Hystricidae & Indian Porcupine & Hystrix indica (Kerr, I792) & Schedule IV \\
Leporidae & Hare & Lepus nigricollis (F. Cuvier, 1823) & Schedule IV \\
Muridae & Common House Rat & Rattus rattus (Linneaus I758) & ScheduleV \\
& 5 striped Palm Squirrel & Funambulus pennanti (Wroughton, 1905) & Schedule IV \\
Sciuridae & Grey Musk Shrew & Suncus murinus (Linneaus I766) & - \\
\hline
\end{tabular}

Table I I List of fishes reported from the study area

\footnotetext{
*not seen directly.
}

\begin{tabular}{|c|c|c|c|}
\hline S.No. & Family & Common name & Scientific name \\
\hline I. & Carangidae & Seer Fish & Caranx atropus \\
\hline 2. & Clupeidae & Hilsa & Tenualosa ilisha \\
\hline 3. & Oxudercidae & Mud Skipper & Gobius boddarti \\
\hline 4. & & Thread Fin & Polynemus indicus \\
\hline 5. & Polynemidae & Indian Salmon* & $\begin{array}{l}\text { Eleutheronema } \\
\text { tetradactylum }\end{array}$ \\
\hline 6. & Sapridae & $\begin{array}{l}\text { Long Spine Sea- } \\
\text { bream* }\end{array}$ & Argyrops spinifer \\
\hline
\end{tabular}




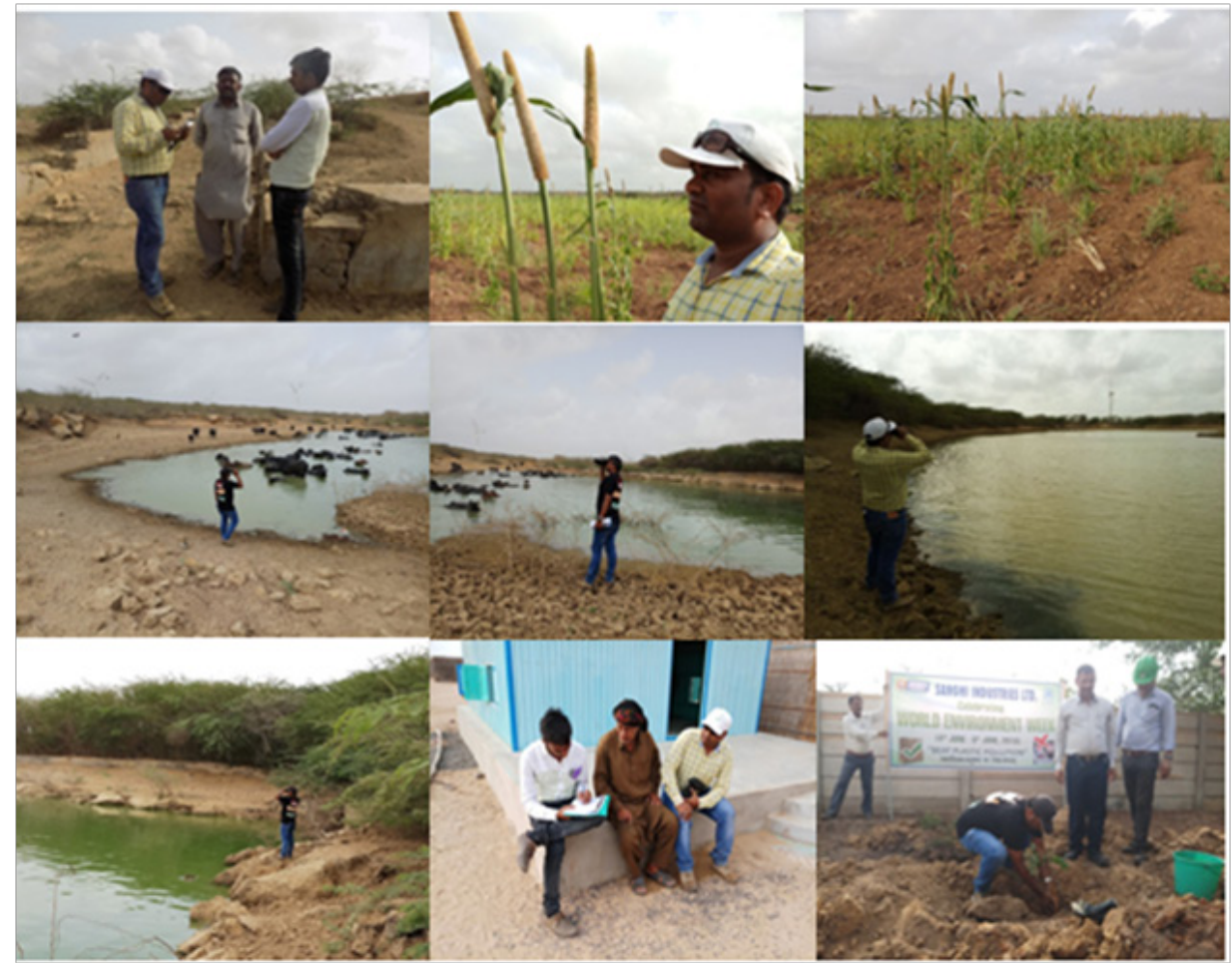

Figure 3 Spotting during primary survey.

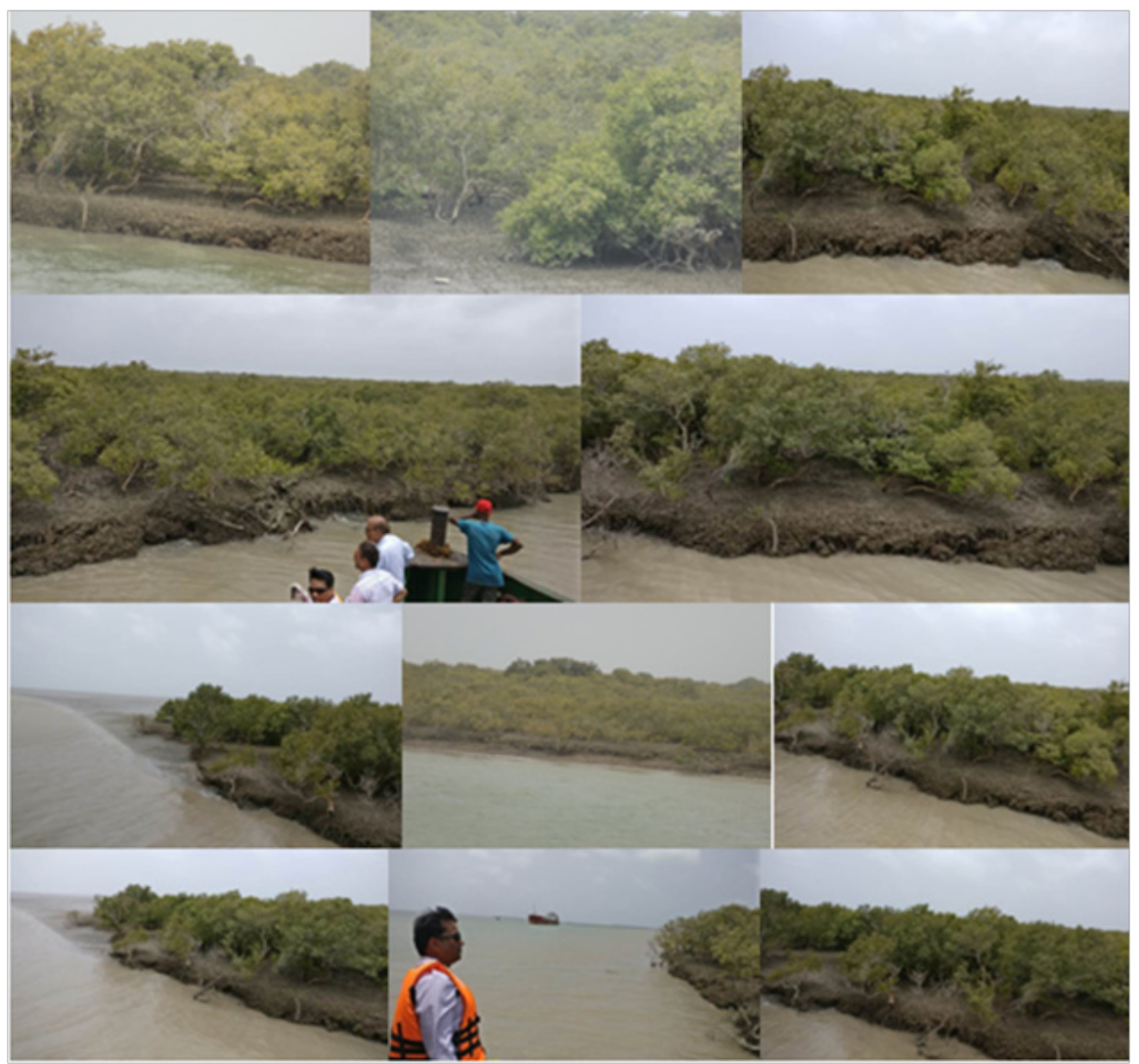

Figure 4 Mangrove in the study area. 


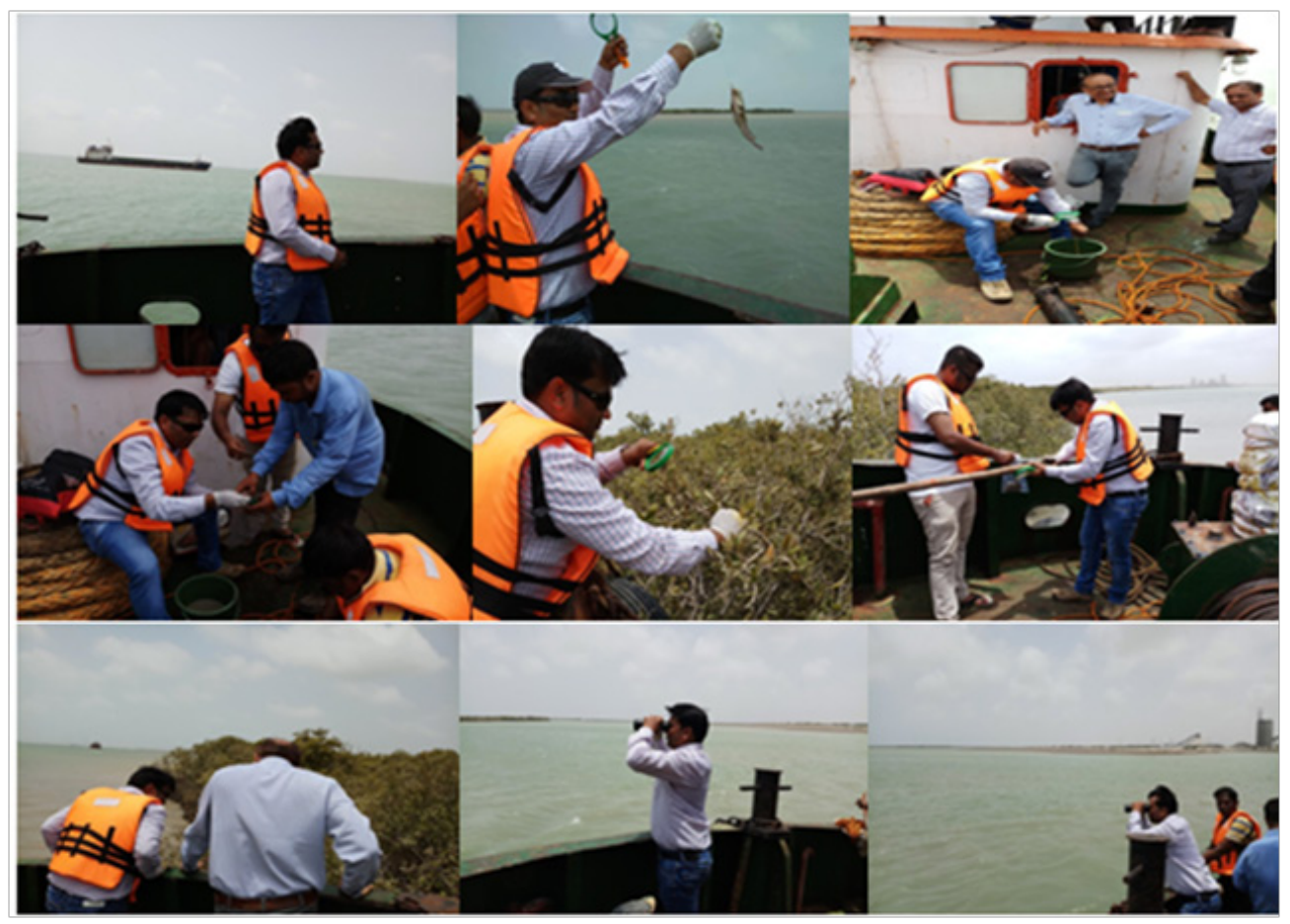

Figure 5 Marine survey.

\section{Conclusion}

The area in question has one thermal power plant running by Sanghi Industries Limited and one Jetty (walkway accessing the centre of an enclosed water body or structure that projects from the land out into water) structure built in 1994-1995 which is mainly used to Cement and other solid cargo handling by Sanghi Industries Limited. Sanghi Industries Limited is among leading cement manufacturers from Western India. Total 34 species of trees belong to 17 families are enumerated from the study area. The dominant trees in the study area are Mangifera indica L (Mango trees) Azadirachta indica (Neem), Plumeria rubra L. (Champa), Babool (Acacia nilotica), Casuarina equisetifolia L. (Casuarina), Dalbergia sisoo L. (Shisham), etc. Total 21 shrub species belong to 15 families are enumerated from the study area. The dominant shrub community in this area was represented by Prosopis juliflora, Calotropis procera, Cereus peruvianus, Xanthium strumarium L., Abelmoschus manihot L., Hibiscus rosa sinensis L., etc. Some part of the study area falls coastal area which is declared as mangrove forest (10.68\%), there is Avecenia marina was observed during the study. Four species of mangrove were encountered from the study area were terrestrial mangrove. The common birds observed in the study area are Accipiter badius, Halcyon coromanda, Anhinga melanogaster, Apus apus, Egretta garzetta, Columba livia, Streptopelia orientalis, Anthus campestris, Motacilla cinerea, Motacilla flava, Nectarinia asiatica, Phalacrocorax fuscicollis, Pycnonotus cafer, Limosa limosa, Platalea ajaja, etc. Common Mongoose (Herpestes edwardsii), Jungle cat were observed which are protected under schedule II and Nilgai (Boselaphus tragocamelus) is Schedule-III animal as per Wildlife Protection Act 1972. The Squirrel, Indian Porcupine, Hare etc. are protected under schedule IV. There is no significant faunal assemblage here except for some insects like Honey Bees (Apis sp.) and Gum leaf Grasshopper (Goniaea australasiae). The study represent that the area in question is not rich in biodiversity, although the marine ecosystem needs to be affected by hot water discharge into sea. Therefore, an urgent need for marine ecosystem management plans to be implemented during operation phase.

\section{Acknowledgements}

None.

\section{Conflict of interest}

Author declares that there is no conflict of interest.

\section{References}

1. Hutto D, Pletsechel SM, Hendrick P. A fixed radius point count method for non-breeding season use. The Auk. 1986;103:593-602.

2. Welsh BHH. Monitoring herpetofauna in woodlands of north western California and south west Oregon: a comparative strategy. AGRIS. 1987.

3. Welsh C, HH Jr, Lind A. The structure of the herpetofaunal assemblage in the Douglas-fir/hardwood forests of northwestern California and south western Oregon. 1991;p. 395-411.

4. Thommpson FID, Davidson IJ, O' Donnell S. et al. Use of track transects to measure the relative occurrence of some arboreal mammals in uncut forest and regeneration stands. Canadian Journal of Zoology. 2019;67:1816-1823.

5. Allen EL, Engeman R, Krupa H. Evaluation of three relative abundance indices for assessing dingo population. Wildlife Research. 1996;23:197206. 
6. Misra R. Ecology Workbook. Scientific Publishers. 2013; p.31-45.

7. Bentham G, Hooker JD. Genera plantarum. L Reeve and Co., London. 1862.

8. BirdLife International. Threatened Birds of the World. Lynx Edicions and BirdLife International. UK: Barcelona and Cambridge; 2000.

9. BirdLife International. Threatened Birds of the World 2004. CD-ROM. UK: BirdLife International, Cambridge; 2004.

10. BirdLife International. State of the World's Birds 2004-Indicators for our changing world. UK: BirdLife International, Cambridge; 2004.

11. BirdLife International. The BirdLife checklist of the birds of the world, with conservation status and taxonomic sources. 2010.

12. Ahmedullah M, Nayar MP. Endemic Plants of the Indian region. Culcutta: Botanical Survey of India. 1987; p. 147.

13. Anderson T. An enumeration of the Indian species of Acanthaceae Journal of Linnaean Society. 1867;9: 425-454.

14. Gamble JS. The Flora of Presidency of Madras 2. Culcutta: Botanical Survey of India; 1924; p.743.

15. IUCN. IUCN Red List Categories. Prepared by the IUCN Species Survival Commission. Switzerland: IUCN, Gland; 1994.

16. IUCN. IUCN Red List Categories and Criteria: Version 3.1. IUCN Species Survival Commission. UK: IUCN, Gland, Switzerland and Cambridge; 2001.

17. IUCN. Guidelines for Application of IUCN Red List Criteria at Regional Levels: Version 3.0. UK: IUCN Species Survival Commission, IUCN, Gland, Switzerland and Cambridge. 2003.

18. IUCN. Red List of Threatened Species. 2008.

19. IUCN. Guidelines for Using the IUCN Red List Categories and Criteria, version 8.1, Standards and Petitions Subcommittee of the IUCN Species Survival Commission; 2010.

20. Ohasi H. Flora of Eastern Himalaya, Third Report. University Museum of University of Tokyo Bulletin. 1975;8:1-458.

21. Jain S.K. Rare and Endangered Specles: Observation on rare, imperfectly known endemic plants. In the sacred groves of Western Maharashtra. Calcutta: Bot Sur of India; 1983;169-178.
22. Jain SK. Dictionary of Indian folk medicine and ethnobotany. New Delhi: Deep publications. 1991.

23. Jain SK. The Problem of Endangered Species. Concepts, Problems and Solutions. In: Tropical Ecosystems: Ecolosv and Management. In: KP Singh, JS Singh, editors. New Delhi: Iiley Eastern iimited; 1992;69-80.

24. Jain SK, Sastry ARK. Threatened plants of India - A State of the Alf Report. New Delhi: Bot Surv of India; 1980.

25. Jain SK, Sastry ARK. Safeguarding Plant diversity in threatened Natural Habitats. In: Conservation of Threatened Natural Habitats. In: Anthony, V Hall, editors. African nat Sci Prog Report. 1984;92.

26. Kumar Ashok. Butterfly (Lepidoptera: Insecta) Diversity from Different Sites of Jhagadia, Ankleshwar, District-Bharuch, Gujarat. Oct Jour Env Res. 2013;1(1):09-18

27. Kumar Ashok. Environmental Management Plan for Chemical Industries Especially Resin Manufacturing Unit. Oct Jour Env Res. 2014;2(3):262273.

28. Kumar Ashok, Aggarwal Savita Goyal. Ecology and Biodiversity status of Sachin gidc and its surroundings with Special reference to Conservation measures for Indian Peafowl (Pavo cristatus) schedule -I Bird species. Oct Jour Env Res. 2013;2(1):82-100.

29. Kumar Ashok, Aggarwal Savita Goyal. Study of Common Property Resources (CPR) With Special Reference To Water And Biological Resources At Projected Area Near Village Ninat, Bardoli, District-Surat. Oct Jour Env Res. 2013;1(4):319-331.

30. Kumar Ashok, Srivastava Meena. Diversity of medicinal Plants in Uttarakhand and their conservation Strategy with special reference to Orchids, In: Proceeding of National Conference on Environementla Health: Challaneges and Management. Lucknow: Pt. Deendayal Upadhyay Govt. PG College Rajajipuram. 2012. 139-142.

31. Kumar Ashok, Srivastava Meena, Goyal Savita. The Biodiversity At Sandi Bird Sanctuary, Hardoi With Special Reference to Migratory Birds. Oct Jour Env Res. 2013;1(3):173-181.

32. Oldfield S, Lusty C, MacKinven A. The World List of Threatened Trees. World Conservation Press, Cambridge. 1998.

33. Shendage SM, SR Yadav. Revision of the Genus Barleria (Acanthaceae) in India. Rheedea. 2010;20(2):81-230. 